\title{
Efficacy of Voriconazole Prophylaxis in Pediatric Patients with Acute Myeloid Leukemia, Single Center Experience, Egypt
}

\author{
Youssef Madney, ${ }^{1,}$, Omar Arafah ${ }^{1}$, Hader Elmahalawy $^{2}$, Lobna Shalby ${ }^{1}$ \\ ${ }^{1}$ Pediatric Oncology Department, National Cancer Institute, Cairo University, Children Cancer Hospital, Cairo, Egypt \\ ${ }^{2}$ Clinical Pathology Department, National Cancer Institute, Cairo University, Cairo, Egypt
}

\section{Email address:}

dryoussef80@hotmail.com (Y. Madney)

${ }^{*}$ Corresponding author

\section{To cite this article:}

Youssef Madney, Omar Arafah, Hader Elmahalawy, Lobna Shalby. Efficacy of Voriconazole Prophylaxis in Pediatric Patients with Acute Myeloid Leukemia, Single Center Experience, Egypt. Cancer Research Journal. Vol. 7, No. 2, 2019, pp. 58-65.

doi: $10.11648 /$ j.crj.20190702.15

Received: May 2, 2019; Accepted: June 11, 2019; Published: June 24, 2019

\begin{abstract}
Patients with hematologic malignancies are at higher risk for invasive fungal infections (IFI) mainly patients with acute myeloid leukemia. Antifungal prophylaxis can help to decrease the incidence of these infections and their related complications. Prospective study compared to historical control data included 136 newly diagnosed Acute Myeloid Leukemia patients treated at the National Cancer Institute, Cairo University from 2011 to 2014. The prospective group received primary Voriconazole compared to retrospective control regarding the infectious complications and incidence of fungal infection. Results showed that one hundred thirty-six (136) newly diagnosed pediatric AML patients were included in the study, 61 patients didn't receive antifungal prophylaxis (Non- prophylactic arm) while 75 patients received voriconazole prophylaxis (prophylactic arm). The median age among both groups was 5.5 years old. Thirty-one (50\%) of the 61 patients in (non prophylactic arm) and five (6.6\%) of the 75 patients enrolled in group B (prophylactic arm) developed an invasive fungal infection. The most commonly affected sites were pulmonary (34/36) while fungal sinus infection was reported in 2 patients. Most patients develop an invasive fungal infection during the induction treatment phase. Primary prophylaxis with voriconazole had a highly statistically significant impact on the reduction of incidence of invasive fungal infection between 2 groups ( $\mathrm{p}$ value.001). Fungal attributable mortality was reported in 8 patients $(13 \%)$ in the historical group (no antifungal prophylaxis) in comparison to 2 patients $(2.6 \%)$ in group patients received voriconazole antifungal prophylaxis. Three Overall and Event-free survival were comparable between both groups. In conclusion, Prophylactic Voriconazole significantly decreased the incidence of fungal infections but it had no impact on diseases or overall survival outcome. Bacterial sepsis and disease-related mortality was the main cause of deaths among our group patients.
\end{abstract}

Keywords: Acute Myeloid Leukemia, Invasive Fungal Infection, Voriconazole

\section{Introduction}

Invasive fungal infections (IFIs) are a serious threat to pediatric patients with hematological malignancies undergoing chemotherapy or hematopoietic stem cell transplantation. In particular, the incidence of IFIs is higher in acute myeloid leukemia (AML) [1]. IFIs have been responsible for considerable morbidity and excessive mortality in this immunocompromised pediatric population $[2,3]$. Early detection of IFIs remains the cornerstone for appropriate management and improved survival. In contrast, nonspecific clinical manifestations make prompt IFI diagnosis challenging, and conventional diagnostic tests for IFI detection in cancer children are either insensitive or need further validation [4]. For these reasons, prevention of their development constitutes an optimal way to improve outcomes of IFIs in pediatric patients. The risk of invasive aspergillosis (IA) is different over all the phases of AML treatment. The majority of AML patients usually experience IA after the first cycle induction of chemotherapy. An IFI 
during the first induction may markedly compromise the following treatment strategy for AML [5]. Antifungal prophylaxis of IFIs may have an essential role in this setting. The availability of new triazoles (i.e., voriconazole, posaconazole) characterized by a broader spectrum may have an essential role for antifungal prophylaxis [6].

The ideal antifungal prophylactic agent should be safe, effective, fungicidal against a broad spectrum of fungal pathogens, available in both oral and intravenous formulation and associated with a low incidence of resistance [6]. These criteria identified triazoles as a beneficial class of oral antifungal drugs, more suitable for chemoprophylaxis of IFIs than Amphotericin- B and other drugs, available only in intravenous (iv) formulation. Voriconazole is a second generation, broad - spectrum triazole with clinical activity against yeasts and molds, including Aspergillus, Candida, Fusarium and Scedosporium species, but not Zygomycetes [7]. Voriconazole has demonstrated safety and efficacy as a first - line treatment for invasive aspergillosis [8] and as first - line treatment of serious Candida infections [9].

We aimed to evaluate the role of voriconazole prophylaxis in decreasing incidence of fungal infection and fungal related mortality among pediatric acute myeloid leukemia patients treated at national cancer institute Egypt during the period from (2011-2014).

\section{Patient and Methods}

\subsection{Patient Population}

This double arms retrospective and the prospective study included all newly diagnosed patients with Acute Myeloid Leukemia who were admitted at pediatric oncology department, National Cancer Institute, Cairo University Egypt, during the time interval between first of January 2011 till the end December 2014. All Patients were followed up till September 2015.

\section{Patients Eligibility Criteria}

Patients below eighteen years old, newly diagnosed patients with Acute Myeloid Leukemia, treated with the COG protocol adopted for treatment of AML pediatric patients.

\subsection{Methods}

Study population was divided into two cohorts: Retrospective (Group A): The retrospective arm included all patients diagnosed during the period between the (2011 2014) and Prospective (Group B): The prospective arm started from the (2013 - 2014).

\subsubsection{Voriconazole Prophylaxis}

For patients participating in the group B protocol, voriconazole prophylaxis was initiated after the induction phase of chemotherapy and continued until neutrophil count recovery, approximately six weeks after the final course of chemotherapy. Voriconazole prophylaxis was given orally at a dose of $200 \mathrm{mg}$ twice a day for patients weighing $40 \mathrm{~kg}$ or more and $100 \mathrm{mg}$ twice a day for those weighing less than $40 \mathrm{~kg}$. In cases when intravenous administration was indicated, voriconazole was administered at $4 \mathrm{mg} / \mathrm{kg} / \mathrm{dose}$ every 12 hours. Therapeutic drug monitoring was not routinely performed.

\subsubsection{Identification and Case Definitions}

Data was extracted from documentation progress notes written in patient files during initial diagnosis and with each cycle chemotherapy and their supportive care admissions. Special attention was given to average duration of delay for each chemotherapy cycle, symptoms and signs of infections, episodes of fever, the timing of the first spike of fever, duration of fever and neutropenia, microbiological, serological data baseline and follow up as well as adjusted antimicrobial and antifungal prophylaxis and therapy. Radiological information has been collected from revision principally of the available films and reports as possible with a comparison between cycles.

\subsection{Definitions of Fungal Infection and Response}

Cases of IMI were selected according to the revised definitions for invasive fungal diseases of the European Organization for Research and Treatment of Cancer/Invasive Fungal Infections Cooperative Group and the National Institute of Allergy and Infectious Diseases Mycoses Study Group Consensus Group (ie, the EORTC/MSG criteria) [10]. The outcome was measured at 12 weeks after the diagnosis of IMI as recommended by the EORTC/MSG therapeutic response and outcome consensus statement. Complete response was defined as resolution of all signs/symptoms attributed to fungal infection, whereas partial response was defined as the improvement of attributable signs and symptoms. For deaths occurring within 12 weeks after diagnosis of IMI, information was collected about the cause of death and its relationship to active fungal disease.

\subsection{Statistical Analysis}

Data were analyzed using IBM SPSS advanced statistics version 22 (SPSS Inc., Chicago, IL). Numerical data were expressed as mean and standard deviation or median and range as appropriate. Qualitative data were expressed as frequency and percentage. Chi-square test or Fisher's exact test was used to examine the relationship between qualitative variables. Survival analysis was done using the Kaplan-Meier method and comparison between two survival curves was done using the log-rank test. All tests were two-tailed. A pvalue $<0.05$ was considered significant. Highly significant at a p-value of 0.001 .

\section{Results}

One hundred thirty-six (136) newly diagnosed pediatric AML patients were included in the study, 61 patients didn't receive antifungal prophylaxis (group $\mathrm{A}=$ Non- prophylactic arm) while 75 patients received voriconazole prophylaxis (group $\mathrm{B}=$ prophylactic arm). The median age among both 
groups was 5.5 years old (range 0.6-17). Males constituted $60 \%$ of the whole cohort versus $40 \%$ female patients. All patient's initial leukemia characteristics are described in (Table 1). Thirty-one $(50 \%)$ of the 61 patients in (Non prophylactic arm) and five $(6.6 \%)$ of the 75 patients enrolled in group B (prophylactic arm) developed an invasive fungal infection. The most commonly affected sites were pulmonary (34/ 36) while fungal sinus infection was reported in 2 patients. Most patients develop an invasive fungal infection during the induction treatment phase, from 36 patients had fungal infection in both groups, 25/36 patients (69\%) developed an invasive fungal infection during induction phase chemotherapy, while six patients (16\%) were diagnosed during intensification therapy. On the other hand, there were only 4 patients $(11 \%)$ and 1 patient $(3 \%)$ developed an invasive fungal infection during induction and intensification therapies respectively in patients of Group B (with primary prophylaxis). Toxicity profile of voriconazole was acceptable, Hypokalemia and febrile reactions were the main toxicity encountered in Group A patients due to excess use of amphotericin B. Skin rash and elevated liver functions occurred more in Group B patients.

Table 1. Clinical and Laboratory Characteristics of Study group Patients.

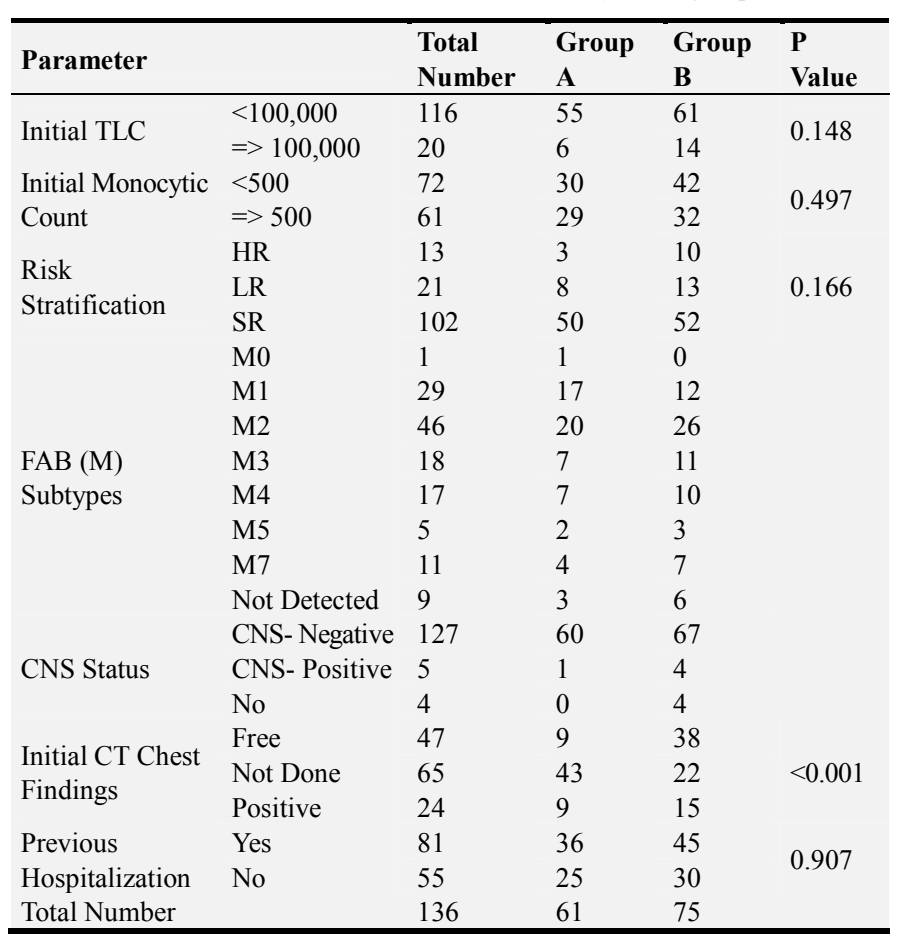

No statistically significant difference was detected between the two groups populations for age or gender. There is no statistically significant difference for Initial TLC, Initial monocytic Count, Risk stratification, previous Hospitalization on the incidence of invasive fungal infections. Patients with inadequate response to treatment have prolonged myelosuppression and they are more prone to develop an invasive fungal infection. In our study, remission status post induction 1 was not statistically significant as risk factor for incidence of fungal infection ( $\mathrm{P}$ value is 0.07). Primary prophylaxis with voriconazole had a highly statistically significant impact on the reduction of incidence of invasive fungal infection between 2 groups (p-value.001) (Table 2).

Table 2. Prognostic factors correlation with the incidence of fungal infections among study group patients.

\begin{tabular}{llll}
\hline & $\begin{array}{l}\text { Group 1 } \\
(\mathbf{N = 6 1 )}\end{array}$ & $\begin{array}{l}\text { Group 2 } \\
(\mathbf{N = 7 5 )}\end{array}$ & $\begin{array}{l}\text { P } \\
\text { value }\end{array}$ \\
\hline $\begin{array}{l}\text { Age (yrs.) Median (Range) } \\
\text { Gender }\end{array}$ & $6.0(0.6-16)$ & $5.0(0.6-17)$ & 0.645 \\
Male & $40(65.6 \%)$ & $41(54.7 \%)$ & 0.197 \\
Female & $21(34.4)$ & $34(45.3)$ & \\
Initial TLC Median (Range) & $13.3(1-65.9)$ & $18.4(1.2-50)$ & 0.103 \\
Monocytic count, Median & $440(0-57100)$ & $387(0-170000)$ & 0.806 \\
(Range) & $25(41 \%)$ & $30(40 \%)$ & 0.907 \\
Previous Hospitalization & & & 0.166 \\
Risk Stratification & $3(4.9 \%)$ & $10(13.3)$ & \\
HR & $8(13.1 \%)$ & $13(7.3)$ & \\
LR & $50(82 \%)$ & $52(69.3)$ & $<0.001$ \\
SR & & & \\
Impact of antifungal prophylaxis & 30 & $70(93.3 \%)$ & \\
Free & $31(50.8 \%)$ & $5(6.7 \%)$ & 0.073 \\
IFD & & & \\
Induction 1 Remission status & $35(57.4 \%)$ & $54(72 \%)$ & \\
In CR & $21(34.4 \%)$ & $13(17.3 \%)$ & $8(10.7 \%)$ \\
Not in CR & $5(8.2 \%)$ & \\
Not Done* & & & \\
\hline
\end{tabular}

1. HR, High risk SR, Standard risk LR, Low risk IFD; invasive Fungal Disease $\mathrm{CR}=$ Complete remission

2. * Not Done; patient died before assessment of leukemia response to treatment

Outcome at 12 weeks after diagnosis of IMI is summarized in (Table 3). The number of patients showing a complete and partial response to therapy at 12 weeks was different in both groups. From 31 patients who developed IMI in Group A, 18 patients showed a complete response, three patients showed partial response while 10 patients had a progressive fungal infection on antifungal therapy, all of the progressive cases shifted to voriconazole as a second-line treatment and had a complete response. In Group B, five (5) patients developed IMI during voriconazole primary prophylaxis. Three patients continued on voriconazole and had complete radiological and clinical remission while two patients had a progressive fungal infection and shifted to Liposomal Amphotericin B. Thirtynine patients $(63 \%)$ died in group A while 47 patient $(62 \%)$ died in group B during the study period. Among patients with IMI, the mortality rate attributable to IMI was reported in 8 patients $(13 \%)$ in Group A (20\% of total deaths in group A) in comparison to 2 patients $(2.6 \%)$ in Group B ( $4 \%$ from total deaths in group B). Disease-related mortality occurs in 13 (21\%) patients in Group A as compared to 17 patients (22\%) in Group B. Bacterial sepsis-related mortality was reported in 18 patients (29\%) in Group A (without levofloxacin prophylaxis) in comparison to 28 patients (37\%) in Group B. In order to exclude any effect of disease on mortality, only patients who died in complete remission (CR) were further analyzed. Five mortalities attributed to fungal infections in Group A who died in CR (8\%) versus one patient in Group B $(4.8 \%)$. 
Table 3. Incidence and IFD Characteristics of all Study group patients.

\begin{tabular}{|c|c|c|c|}
\hline Total study groups $=136$ & Group A (Non- prophylactic arm) = 61 Patients & Group B $($ Prophylactic arm) $=75$ & P-value \\
\hline $\begin{array}{l}\text { Incidence of IFD } \\
\text { Timing }\end{array}$ & $31(50 \%)$ & $5(6 \%)$ & $<0.001$ \\
\hline Induction & $25(80.6)$ & $4(80)$ & $<01$ \\
\hline Intensification & $6(19.4)$ & $1(20)$ & \\
\hline Organ System Involvement & & & 0.262 \\
\hline Pulmonary & $30(96.8)$ & $4(80)$ & 0.202 \\
\hline Sino nasal & $1(3.2)$ & $1(20)$ & \\
\hline Definition of IFD (EORTC*) & & & 0.262 \\
\hline Possible & $30(96.8)$ & $4(80)$ & \\
\hline Probable & $0(0)$ & $0(0)$ & \\
\hline Proven & $1(3.2)$ & $1(20)$ & \\
\hline Response to treatment** & & & 1.000 \\
\hline Complete remission & $18(58.1)$ & $3(60 \%)$ & \\
\hline Partial remission & $3(9.7)$ & $0(0 \%)$ & \\
\hline Progressive & $10(32.3)$ & $2(40 \%)$ & \\
\hline All causes related mortality & $39(63.9)$ & $47(62.7)$ & 0.879 \\
\hline Mortality Causes & & & 0.060 \\
\hline Fungal attributable cause of mortality & $8(20.5)$ & $2(4.3)$ & \\
\hline Bacterial gram-negative sepsis & $18(46.2)$ & $28(59.6)$ & \\
\hline Disease related mortality & $13(33.3)$ & $17(36.2)$ & \\
\hline
\end{tabular}

*EORTC $=$ Revised definitions of invasive fungal disease from the European Organization for Research and Treatment of Cancer/Invasive Fungal Infections Cooperative Group and the National Institute of Allergy and Infectious Diseases Mycoses Study Group (EORTC/MSG) [10] **Defining Responses to Therapy and Study Outcomes in Clinical Trials of Invasive Fungal Diseases: Mycoses Study Group and European Organization for Research and Treatment of Cancer Consensus Criteria [25].

With the median duration of follow up of 12 months, the overall survival and event-free survival for the whole Group of patients at three years were $37.5 \%$ and $34.5 \%$ respectively (Figure 1). While assessing the impact of voriconazole primary prophylaxis on survival outcome, it was found that the event-free survival of Group A-patients (without prophylaxis) at three years was $36.6 \%$ compared to $32.5 \%$ in Group B patients $(\mathrm{P}$-value $=0.65)$. Also, the 3 -years overall survival of Group A patients (without prophylaxis) was $38.1 \%$ compared to $36 \%$ in Group B patients (P-value $=$ 0.757) (Figure 2).

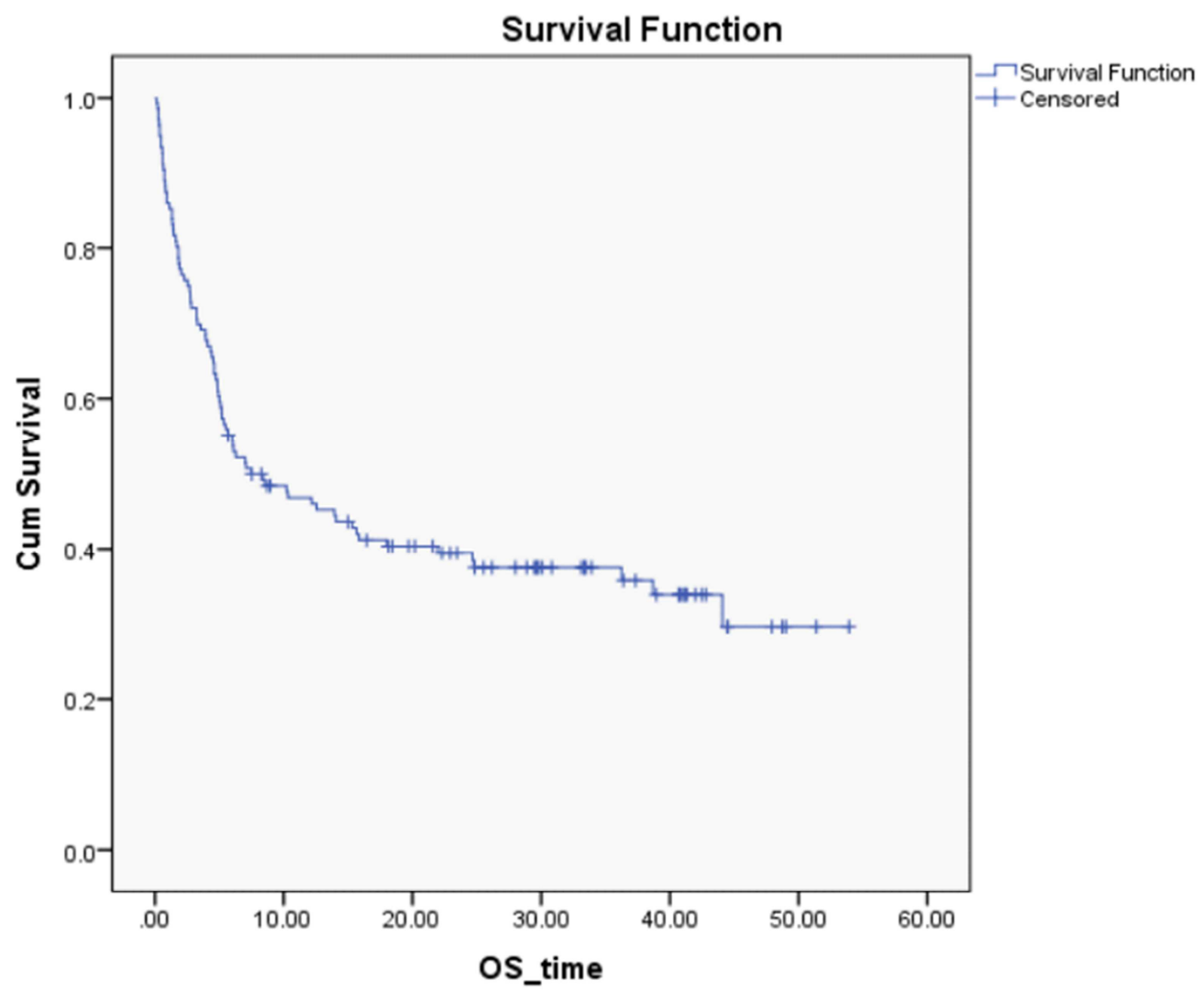




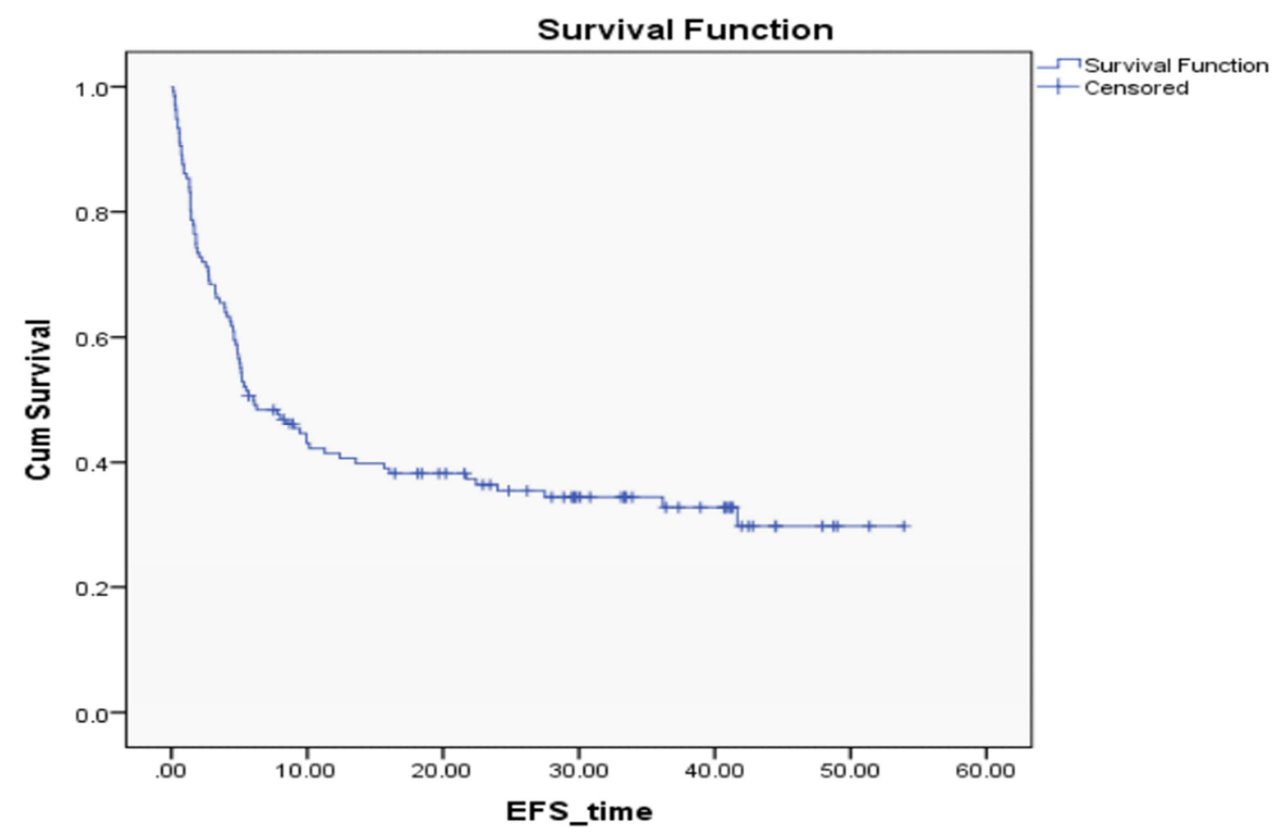

Figure 1. Overall survival and EFS of the study group patients.
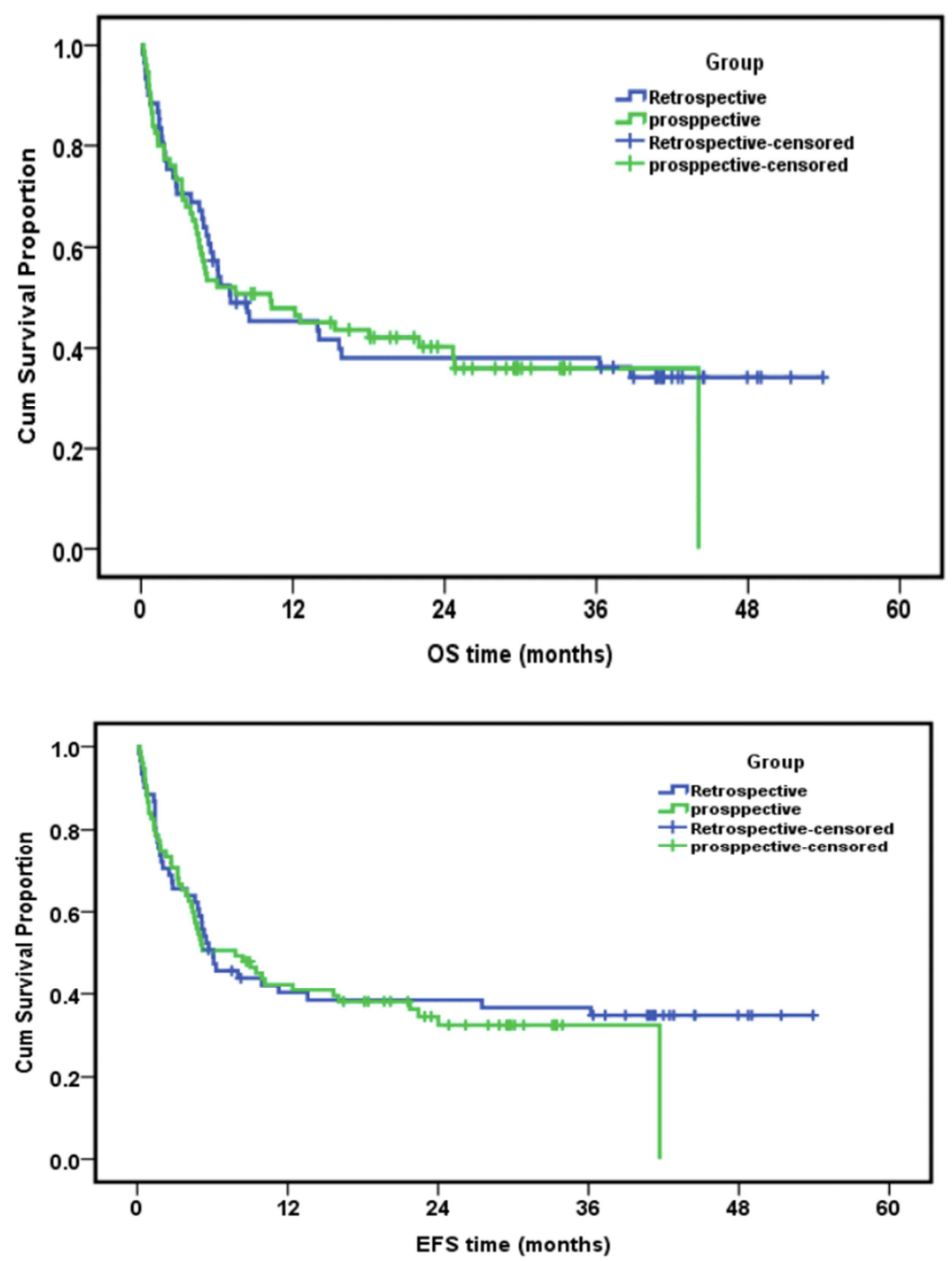

Figure 2. Impact of antifungal prophylaxis on OS and EFS. 


\section{Discussion}

Invasive fungal infections (IFIs) occur in 5\% - 40\% of patients with hematologic malignancies most commonly in AML [11]. Aspergillus and Candida species account for most of all cases. The epidemiology of IFIs evolves under the selection pressure of antimicrobials [12,13]. IFIs constitute a leading cause of morbidity and mortality as increasing use of intensively immunosuppressive cancer therapies. IFIs represent an important reason for delays and reductions of antileukemia treatments, they can also reduce AML cure rates $[14,15]$. High mortality from IFIs is due to difficult diagnosis and delayed treatment initiation, the limited activity of antifungal agents, drug side effects, and increasing use of high-dose corticosteroids [16]. Primary prevention of fungal infections demonstrated to reduce IFIs as well as infection- attributable and all-cause mortality $[17,18]$.

In this study, we compared the incidence and outcome of IMI for patients participating in the treatment protocol for AML; Study population was divided into two arms retrospective arm used no antifungal prophylaxis (From 2011 to 2012), and prospective arm (group B) used voriconazole prophylaxis (From 2013 to 2014). Our findings demonstrate the changing incidence of IMI at our institution after voriconazole prophylaxis was established as part of the standard supportive care of patients with AML. Before the use of voriconazole prophylaxis at our institution, $50 \%$ of AML patients had an invasive fungal disease in comparison to $6 \%$ incidence of IMI after voriconazole prophylaxis. Gabriela et al. [19] supported the decreased incidence of invasive aspergillosis after use of voriconazole prophylaxis and demonstrated changing in the epidemiology of IMI at their institution after voriconazole prophylaxis was established as part of the standard supportive care of patients with AML without significant increase in the incidence of zygomycosis associated with routine use of voriconazole prophylaxis [19].

Most invasive fungal infection in this study occurred during the induction phase of chemotherapy with a highly significant statistical value ( $p$ value $<01$ ). It is well accepted that prolonged and profound neutropenia, typically seen in the first 30 days after induction chemotherapy, is associated with IFIs [20]. In Ursula et al. study, after day 15, the predominant causes of death were complications caused by infections, particularly bacterial and fungal infections. The incidence of lethal infections was highest during induction therapy and decreased after that [21].

There have been concerns that widespread use of voriconazole prophylaxis, while effective in preventing some fungal infections, may lead to an increase in fungal infections for which voriconazole is not an effective treatment. Voriconazole is not active against the zygomycetes; several studies have reported the emergence of zygomycosis in adult HSCT and AML patients who received prophylaxis with voriconazole [22-24]. In our study, from 75 patients under voriconazole prophylaxis, only 6 patients had developed IFD (one patient proved to be invasive aspergillosis by histopathology), we did not see a significant increase in cases of zygomycosis keeping in mind a small number of patients and lacking diagnostic tools for biopsy and histopathological identification (only 2 patients had biopsy for diagnosis of IFD).

Response assessment for fungal infection done by EORTC response criteria at 12 weeks after diagnosis of IMI using clinical and radiological tools [25]. From 31 patients who developed IMI in Group A, 18 patients showed a complete response; three patients showed partial response while 10 patients had a progressive fungal infection on antifungal therapy (liposomal amphotericin B), all of the progressive cases shifted to voriconazole as a second-line treatment and had a complete response. In Group B (prophylactic arm) Breakthrough of fungal infections on top of voriconazole prophylaxis was reported in 5 patients, three patients continued on voriconazole (No available therapeutic drug monitoring for voriconazole) and had complete radiological and clinical remission while two patients had a progressive fungal infection and shifted to Liposomal Amphotericin B.

Higher mortality rate was observed among our study group $(86 / 136(63 \%))$, the mortality rate in non- prophylactic arm was $63 \%$ (39/61patients) while it was $62 \%$ in prophylactic arm (47/75 patients). Bacterial sepsis was the leading cause of mortality $(53.5 \%)$, disease-related mortality was $(35 \%)$ and fungal related mortality was (11.5\%) among the whole studied group patients. Mortality subgroup analysis between both groups showed that fungal related mortality was $13 \%$ $(8 / 39)$ in non-prophylactic arm patients and $2.6 \%(2 / 75)$ in voriconazole prophylactic arm patients, while bacterial sepsis was the main cause of death in $29 \%$ in non-prophylactic arm while it was the main cause of death in $37 \%$ in prophylactic arm. Disease-related mortality in the non-prophylactic arm was $21 \%$, and it was $22 \%$ in the prophylactic arm group. Primary prophylaxis with voriconazole had marked effect on reduction of fungal infection-related mortality between 2 groups.

The overall survival and event-free survival for the whole Group of patients at three years were $37.5 \%$ and $34.5 \%$ respectively with a median duration of follow up of 12 months. No statistically significant difference was detected between the two groups populations for age or gender. There is no statistically significant difference for Initial TLC, Initial monocytic Count, Risk stratification, previous Hospitalization on the incidence of invasive fungal infections. Inadequate response to treatment and state of leukemia disease had near statically significant (0.08\%) impact on the rate of fungal of infections among both groups which can be explained by more prolonged duration of neutropenia and neutrophil dysfunction as disease effect which can lead to higher risk of fungal infection. The only prognostic factor that had a statistically significant impact was anti-fungal prophylaxis as Primary prophylaxis with voriconazole had a highly statistically significant effect on 
the reduction of incidence of invasive fungal infection between 2 groups ( $\mathrm{P}$-value $=0.001)$.

Our study had many limitations, lack of diagnostic tools for confirmation of the diagnosis of fungal infections like galactomannan antigen as a marker of invasive aspergillosis or B-d glucan as pan-fungal marker, being area of limited resources and lack of support of biomarker tools, clinical and radiological diagnosis was the main standard diagnostic tool for IFD diagnosis. Biopsy for histopathology and microbiological identification is very difficult in our center as lack of tools with the poor general condition of AML patients with high risk of bleeding as severe thrombocytopenia. Lack of diagnostic tools had an impact on true incidence of fungal infection as radiology is only suggestive but not diagnostic for fungal infection. Nonculture diagnostic markers can be helpful for early diagnosis and treatment.

\section{Conclusion}

This study highlighted the impact of antifungal prophylaxis among newly diagnosed AML patients as a higher incidence of IFD (50\% of patients) if no antifungal prophylaxis used. Despite the use of antifungal prophylaxis decrease incidence of antifungal infection, it had no impact on diseases or overall survival outcome. Bacterial sepsis and disease-related mortality was the primary cause of deaths among our group patients which mandate strict guidelines for febrile neutropenia protocols and modifying empirical antibiotic treatment based (de-escalation approach) on local epidemiology of our center as a higher incidence of gramnegative resistance bacteremia.

\section{References}

[1] Caira M, Girmenia C, Fadda RM, Mitra ME, Picardi M, Van Lint MT, et al. Invasive fungal infections in patients with acute myeloid leukemia and in those submitted to allogeneic hemopoietic stem cell transplant: who is at highest risk? Eur J Haematol 2008. doi:10.1111/j.1600-0609.2008.01096. x.

[2] Rosen GP, Nielsen K, Glenn S, Abelson J, Deville J, Moore TB. Invasive fungal infections in pediatric oncology patients: 11-Year experience at a single institution. J Pediatr Hematol Oncol 2005. doi:10.1097/01.mph.0000155861.38641.ca.

[3] Steinbach WJ, Walsh TJ. Mycoses in Pediatric Patients. Infect Dis Clin North Am 2006; 20: 663-78. doi:10.1016/j.idc.2006.06.006.

[4] Pana ZD, Vikelouda K, Roilides E. Diagnosis of invasive fungal diseases in pediatric patients. Expert Rev Anti Infect Ther 2016; 14:1203-13. doi: $10.1080 / 14787210.2016 .1242413$.

[5] L. P, M. C, A. C, M. O, B. M, G. S, et al. Invasive aspergillosis in patients with acute myeloid leukemia: A SEIFEM-2008 registry study. Haematologica 2010.

[6] Pagano L, Caira M. The role of primary antifungal prophylaxis in patients with haematological malignancies.
Clin Microbiol Infect 2014. doi:10.1111/1469-0691.12464.

[7] Marks DI, Pagliuca A, Kibbler CC, Glasmacher A, Heussel $\mathrm{CP}$, Kantecki M, et al. Voriconazole versus itraconazole for antifungal prophylaxis following allogeneic haematopoietic stem-cell transplantation. $\mathrm{Br} \mathrm{J}$ Haematol 2011. doi:10.1111/j.1365-2141.2011.08838. x.

[8] Voriconazole versus Amphotericin B for Invasive Aspergillosis. N $\quad$ Engl J 2002. doi:10.1056/nejm200212193472518.

[9] B. J. K, J. D. S, M. R, P. G. P, C. V, J. H. R, et al. Voriconazole versus a regimen of amphotericin B followed by fluconazole for candidaemia in non-neutropenic patients: A randomised non-inferiority trial. Lancet 2005. doi:10.1016/S0140-6736 (05) 67490-9.

[10] De Pauw B, Walsh TJ, Donnelly JP, Stevens DA, Edwards JE, Calandra $T$, et al. Revised Definitions of Invasive Fungal Disease from the European Organization for Research and Treatment of Cancer/Invasive Fungal Infections Cooperative Group and the National Institute of Allergy and Infectious Diseases Mycoses Study Group (EORTC/MSG) C. Clin Infect Dis 2008; 46: 1813-21. doi:10.1086/588660.

[11] Pechlivanoglou P, Le HH, Daenen S, Snowden JA, Postma MJ. Mixed treatment comparison of prophylaxis against invasive fungal infections in neutropenic patients receiving therapy for haematological malignancies: A systematic review. J Antimicrob Chemother 2014. doi:10.1093/jac/dkt329.

[12] Leventakos K, Lewis RE, Kontoyiannis DP. Fungal Infections in Leukemia Patients: How Do We Prevent and Treat Them? Clin Infect Dis 2010. doi:10.1086/649879.

[13] Tacke D, Buchheidt D, Karthaus M, Krause SW, Maschmeyer G, Neumann S, et al. Primary prophylaxis of invasive fungal infections in patients with haematologic malignancies. 2014 Update of the recommendations of the Infectious Diseases Working Party of the German Society for Haematology and Oncology. Ann Hematol 2014. doi:10.1007/s00277-014-2108-y.

[14] Kontoyiannis DP, Marr KA, Park BJ, Alexander BD, Anaissie EJ, Walsh TJ, et al. Prospective Surveillance for Invasive Fungal Infections in Hematopoietic Stem Cell Transplant Recipients, 2001-2006: Overview of the Transplant Associated Infection Surveillance Network (TRANSNET) Database. Clin Infect Dis 2010. doi:10.1086/651263.

[15] Even C, Bastuji-Garin S, Hicheri Y, Pautas C, Botterel F, Maury S, et al. Impact of invasive fungal disease on the chemotherapy schedule and event-free survival in acute leukemia patients who survived fungal disease: A case-control study. Haematologica 2011.

doi:10.3324/haematol.2010.030825.

[16] Lewis RE, Cahyame-Zuniga L, Leventakos K, Chamilos G, Ben-Ami R, Tamboli P, et al. Epidemiology and sites of involvement of invasive fungal infections in patients with haematological malignancies: A 20-year autopsy study. Mycoses 2013. doi:10.1111/myc.12081.

[17] Ethier MC, Science M, Beyene J, Briel M, Lehrnbecher T, Sung L. Mould-active compared with fluconazole prophylaxis to prevent invasive fungal diseases in cancer patients receiving chemotherapy or haematopoietic stem-cell transplantation: A systematic review and meta-analysis of randomised controlled trials. $\mathrm{Br} \mathrm{J}$ Cancer 2012. doi:10.1038/bjc.2012.147. 
[18] Robenshtok E, Gafter-Gvili A, Goldberg E, Weinberger M, Yeshurun M, Leibovici L, et al. Antifungal prophylaxis in cancer patients after chemotherapy or hematopoietic stem-cell transplantation: Systematic review and meta-analysis. J Clin Oncol 2007. doi:10.1200/JCO.2007.12.3851

[19] Maron GM, Hayden RT, Rodriguez A, Rubnitz JE, Flynn PM, Shenep JL, et al. Voriconazole prophylaxis in children with cancer: Changing outcomes and epidemiology of fungal infections. Pediatr Infect Dis J 2013. doi:10.1097/INF.0b013e3182a74233.

[20] Alison G. Freifeld, Eric J. Bow, Kent A. Sepkowitz, Michael J. Boeckh, James I. Ito, Craig A. Mullen, Issam I. Raad, Kenneth V. Rolston, Jo-Anne H. Young and JRW. Clinical Practice Guideline for the Use of Antimicrobial Agents in Neutropenic Patients with Cancer: 2010 Update by the Infectious Diseases Society of America. Clin Infect Dis 2011. doi:10.1097/IPC.0b013e31822c8f36.

[21] Creutzig U, Zimmermann M, Reinhardt D, Dworzak M, Stary J, Lehrnbecher T. Early deaths and treatment-related mortality in children undergoing therapy for acute myeloid leukemia: Analysis of the multicenter clinical trials AML-BFM 93 and AML-BFM 98. J Clin Oncol 2004. doi:10.1200/JCO.2004.01.191.
[22] Imhof A, Balajee SA, Fredricks DN, Englund JA, Marr KA. Breakthrough Fungal Infections in Stem Cell Transplant Recipients Receiving Voriconazole. Clin Infect Dis 2004. doi:10.1086/423274.

[23] Trifilio S, Singhal S, Williams S, Frankfurt O, Gordon L, Evens A, et al. Breakthrough fungal infections after allogeneic hematopoietic stem cell transplantation in patients on prophylactic voriconazole. Bone Marrow Transplant 2007. doi:10.1038/sj.bmt.1705754.

[24] Y. M, R. K, H. A-M, N. A, H. T, I. Z, et al. "mucormycosis" the emerging global threat; Overview and treatment outcome among pediatric cancer patients in Egypt. Blood 2017.

[25] Segal BH, Herbrecht R, Stevens DA, Ostrosky - Zeichner L, Sobel J, Viscoli C, et al. Defining Responses to Therapy and Study Outcomes in Clinical Trials of Invasive Fungal Diseases: Mycoses Study Group and European Organization for Research and Treatment of Cancer Consensus Criteria. Clin Infect Dis 2008. doi:10.1086/590566. 